\title{
Steuerung und Management in einem Verkehrssystem mit autonomen Fahrzeugen
}

Peter Wagner

\section{Inhaltsverzeichnis}

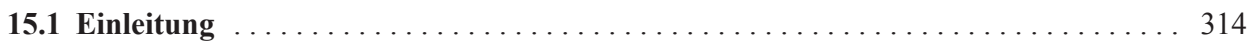

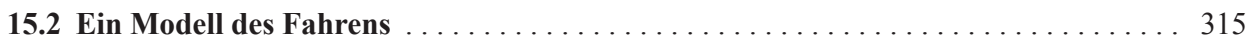

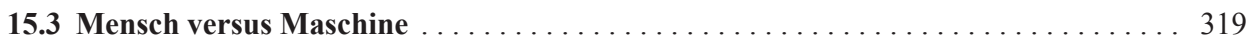

15.4 Anfahren an einer Lichtsignalanlage (LSA) $\ldots \ldots \ldots \ldots \ldots \ldots \ldots \ldots \ldots \ldots \ldots \ldots$

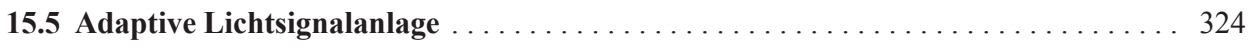

15.6 Grüne Welle mit autonomen Fahrzeugen $\ldots \ldots \ldots \ldots \ldots \ldots \ldots \ldots \ldots \ldots \ldots \ldots \ldots \ldots \ldots \ldots$

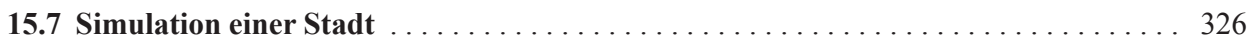

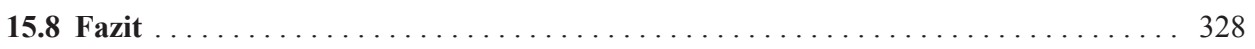

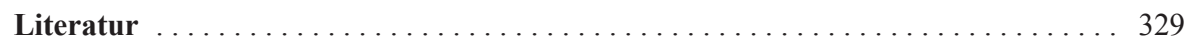

P. Wagner $(\square)$

Deutsches Zentrum für Luft- und Raumfahrt (DLR), Institut für Verkehrssystemtechnik, Deutschland

Peter.wagner@dlr.de 


\subsection{Einleitung}

Dieser Beitrag hat zum Ziel, die Auswirkungen des autonomen Fahrens auf der Ebene des Verkehrsmanagements zu quantifizieren. Dazu wird eine Modellierung des autonomen Fahrens entwickelt, die es ermöglicht, von Menschen geführte und autonome Fahrzeuge mit nur geringen Modifikationen zu verwenden. Das ist wichtig, wenn es darum geht, wie die Instrumente des Verkehrsmanagements weiterzuentwickeln sind, um autonome Fahrzeuge im Verkehrssystem berücksichtigen zu können. Interessant ist in diesem Zusammenhang vor allem der sogenannte gemischte Verkehr, bei dem normale und autonom fahrende Fahrzeuge miteinander agieren. Das dürfte - auch nach der Markteinführung autonomer Fahrzeuge - noch lange der Normalzustand auf den Straßen sein; von daher ist es von großer praktischer Bedeutung, genau diesen Zustand gut zu verstehen, um eventuell auftretende systemische Effekte im Voraus zu erkennen und diesen entgegenwirken zu können.

Da es bislang autonome Fahrzeuge noch nicht gibt, sind Teile der folgenden Betrachtungen und Szenarien sicher spekulativ. Allerdings ist auch die Modellierung des humanen Fahrers noch bei Weitem nicht abgeschlossen, sodass in diesem Beitrag der Schwerpunkt auf einer konsistenten Modellierung liegen wird. Zielrichtung der hier vorgestellten Modellierung ist es, von Menschen geführte und autonome Fahrzeuge möglichst mit dem gleichen Modell zu beschreiben, bei dem sich nur die Parametrierungen unterscheiden. Ein gutes Beispiel dafür ist der einzuhaltende Abstand zum vorausfahrenden Fahrzeug, ausgedrückt durch die Zeitlücke: Ein autonomes Fahrzeug kann hier Werte von 0,3...0,5 s [1] erreichen, von Menschen geführte Fahrzeuge dürfen nach dem Gesetz 0,9 s nicht unterschreiten (in Deutschland). Die entsprechende gesetzliche Empfehlung liegt sogar bei 2,0 s, ein Wert, der aber nur in Zeiten schwacher Verkehrsnachfrage eingehalten wird. Im dichten Verkehr wird dieser Wert zum Teil deutlich unterschritten, als häufigster Wert auf einer dicht befahrenen Autobahn werden 1,1 s erreicht (s. Abb. 15.3), bei einem Durchschnittswert von 1,4 s. Würden die Fahrer sich an die gesetzlichen Vorgaben halten, dann würde der Verkehr auf vielen Straßen sehr viel früher zum Erliegen kommen als derzeit.

Dieser Beitrag ergänzt Kap. 16 und Kap. 19 in diesem Buch. Während Kap. 16 ganz allgemein die verkehrliche Wirkung autonomer Fahrzeuge beschreibt, geht es in diesem Beitrag neben der Modellierung dieser und von Menschen gefahrener Fahrzeuge um Effekte autonomer Fahrzeuge auf das Verkehrsmanagement. Kapitel 19 hingegen ignoriert Fragen des Verkehrsablaufes und der Verkehrssteuerung weitgehend und betrachtet vor allem eine optimale Allokation von Angebot zur Nachfrage unter der Voraussetzung, dass Fahrzeuge geteilt werden können. Ganz richtig kann man an dieser Stelle festhalten, dass eine Kombination dieser Ansätze, zusammen mit einer korrekten Beschreibung des Anteils von Reisenden, die sich von einem robotischen Mobility-on-demand-System transportieren lassen, die bestmögliche Abschätzung des Potenzials autonomer Fahrzeuge ermöglichen würde.

Ebenfalls nicht betrachtet werden hier Effekte, die aus einer grundlegend anderen Organisation des Verkehrs resultieren. Beispielhaft sei hier nur das EU-Projekt CityMobil genannt, in dem solche Szenarien diskutiert und näher untersucht wurden [2]. 
In diesem Beitrag wird an einigen wenigen Beispielen, die nicht vollständig spezifiziert sind, der Frage nachgegangen, wie sich autonome Fahrzeuge auf typische Verkehrsmanagementanwendungen auswirken. Das sind, nach aufsteigender Komplexität geordnet, die Simulation einer einzelnen Lichtsignalanlage (Abschn. 15.4), die Simulation einer Kreuzung, die von einer adaptiven Lichtsignalanlage gesteuert wird (Abschn. 15.5), die Simulation einer grünen Welle (Abschn. 15.6) und die Simulation einer ganzen Stadt (Abschn. 15.7).

Ein Teil der hier zu betrachtenden Fragestellungen kann auf Ergebnisse zurückgreifen, die das Einführen einer intelligenten Abstandsregelung (autonomous intelligent speed control - AIC) auf den Verkehrsablauf vor allem aufAutobahnen hat [3]. Zu diesem Bereich gibt es umfangreiche Literatur, die Dissertation von Kersting [3] und Teile des Buches von Winner et al. [12] geben hier mehr Überblick als in diesem Kapitel möglich ist.

Ein solches AIC-Szenario hat starke Ähnlichkeit zu Use-Case 1 ,„Interstate Pilot with Availability through Driver", und das ist wiederum (aus Sicht des Verkehrsablaufes) ein Spezialfall von Use-Case 3 ,Full Automation with Availability through Driver” (s. Kap. 2 für die Definition der Use-Cases). Das ist auch der Use-Case, der in diesem Kapitel die wichtigste Rolle spielt - wobei es aus Sicht des Verkehrsflusses ziemlich gleichgültig ist, ob der Fahrer verfügbar ist oder nicht. Die Verfügbarkeit des Fahrers könnte wichtig sein, wenn der Einfluss von Ausfällen auf den Verkehrsfluss untersucht wird, dieses Thema wird allerdings in diesem Buch nicht aufgegriffen. Es würde eine detaillierte Statistik voraussetzen, wie häufig so etwas passiert und unter welchen Randbedingungen - eine Information, die beim derzeitigen Stand der Technik autonomer Fahrzeuge nicht verfügbar ist. Die Use-Cases 2 (Valet-Parken) und 4 (Vehicle-on-Demand) spielen in diesem Abschnitt eine untergeordnete Rolle, der Use-Case 4 ist aus Verkehrsflusssicht allerdings wie UseCase 3 zu behandeln. Use-Case 2 wäre interessant, weil er Einfluss hat auf den sogenannten Parksuchverkehr und damit indirekt auf die Verkehrsnachfrage und somit auch die Verkehrssteuerung beeinflusst, würde aber auf der Ebene des Verkehrsmanagements eine deutlich kompliziertere Vorgehensweise voraussetzen als hier geleistet werden kann - so bräuchte es eine präzise Quantifizierung des Parksuchverkehrs in einer Stadt. Auch die in Abschn. 15.7 beschriebene Simulation der Stadt Braunschweig geht davon aus, dass Fahrzeuge, die ihr Ziel erreicht haben, immer und sofort einen Parkplatz finden.

\subsection{Ein Modell des Fahrens}

Modelle, die beschreiben, wie ein Mensch ein Fahrzeug führt, gibt es schon sehr lange [4]. Sehr viele dieser Modelle (für einen Überblick s. [5], [6], [7], [8]) - seit 1950 sind wohl mehr als 100 Modelle allein für den Prozess des Folgens eines vorausfahrenden Fahrzeuges beschrieben worden - lassen sich ohne Weiteres auch als Modelle für ein autonomes Fahrzeug verstehen - wie bereits in Abschn. 15.1 begründet, mit verschiedenen Parametrierungen für Mensch bzw. Maschine. Damit wird es konzeptionell recht einfach, auch gemischten Verkehr zu modellieren und die Auswirkungen auf das gesamte Verkehrssystem zu quantifizieren. 


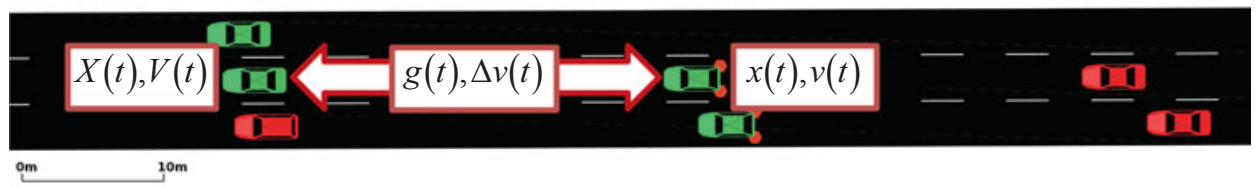

Abb. 15.1 Visualisierung der verwendeten dynamischen Größen anhand eines SUMO [17]-Screenshots. Fahrtrichtung ist von rechts nach links

Im Folgenden liegt der Fokus auf dem Prozess des Fahrzeugfolgens, welches den wichtigsten, aber nicht den einzigen relevanten Prozess darstellt, der darüber bestimmt, wie sich der Verkehrsablauf auf Straßen entwickelt.

Jedes Fahrzeug wird dabei beschrieben durch seine Position $x(t)$, die von der Zeit $t$ abhängt und in Bezug auf eine Referenz (z. B. Anfang des aktuellen Straßenabschnitts) definiert ist, durch seine Geschwindigkeit $v(t)$ und seine Beschleunigung $a(t)$ (s. Abb. 15.1). Bei Mehrspurverkehr kommt noch die Spur hinzu, auf der das Fahrzeug sich befindet, oder die sogenannte Lateralkoordinate, also der Abstand des Fahrzeugs vom Fahrbahnrand. Genau genommen müsste auch noch eine Indizierung jedes Fahrzeugs eingeführt werden, was im Folgenden durch die Beschreibung des vorausfahrenden Fahrzeugs mit großen Buchstaben $X(t), V(t), A(t)$ umgangen wird. Mit den zusätzlichen Variablen Abstand $g(t)=X(t)-x(t)-\ell$ und Geschwindigkeitsdifferenz $\Delta v(t)$ (s. Abb. 15.1) kann dann die Reaktion des Folgefahrzeugs definiert werden als die Beschleunigung, die das Fahrzeug in einer gegebenen Situation appliziert:

$$
a=\frac{d}{d t} v=\dot{v}=f(v, g, \Delta v)
$$

Diese abstrakte Gleichung (15.1) ließe sich noch weiter abstrahieren, z. B. fehlt hier ein Modell für Fahrerfehler und für Fluktuationen ebenso wie die Modellierung einer Reaktionszeit. Ein entsprechendes Fehlermodell wird im Abschn. 15.3 vorgestellt, die Reaktionszeit bleibt ganz außen vor, weil sie ein enorm schwieriges Konstrukt ist: In Messdaten zeigt sich zwar sehr oft, dass die Beschleunigung eines Folgefahrzeugs rund zwei Sekunden der Beschleunigung des vorausfahrenden Fahrzeugs hinterherhinkt, es gibt aber auch Fälle, in denen das Folgefahrzeug rund eine Sekunde vor dem Führungsfahrzeug zu bremsen beginnt - z. B. bei der Annäherung an eine Lichtsignalanlage (Ampel). Im Folgenden soll die abstrakte Gleichung (15.1) genauer spezifiziert werden. Beispielsweise ist es eine wichtige Frage für die folgenden Betrachtungen, wie genau ein autonomes Fahrzeug sich bewegt. Überraschenderweise funktionieren viele der aktuellen adaptiven Abstandsregler und auch veröffentlichte Steuerungsalgorithmen für automatische Fahrzeuge [9], [10], [11] als linearer Regler:

$$
\dot{v}=\alpha\left(g-g^{*}(v)\right)+\beta \Delta v
$$


Typische Parameter für die beiden Zeitkonstanten sind dabei durch $\alpha=1 / 201 / \mathrm{s}^{2}$ und $\beta=1 / 1,51 /$ s gegeben; bei diesen Werten sind solche Regler so eingestellt, dass sie von Fahrern als angenehm und natürlich empfunden werden [12]. Für den bevorzugten Abstand $g^{*}(v)=v \tau$ wird im Wesentlichen die gesetzliche Regel übernommen, wenn auch mit einem etwas kleineren Wert der bevorzugten Zeitlücke $\tau$, z.B. $\tau=1,5 \mathrm{~s}$, der auch im Folgenden verwendet wird. Das Modell in Gleichung (15.2) wurde ursprünglich von Helly 1959 [13] als ein Modell eingeführt, das einen menschlichen Fahrer beschreiben soll. Das unterstreicht die Behauptung, dass viele Fahrermodelle und die Modelle für das autonome Fahren mathematisch sehr ähnlich sind. Worin sie sich unterscheiden, wird versucht in Abschn. 15.3 zu spezifizieren.

Das Modell in Gleichung (15.2) hat Grenzen. Beispielsweise ist es nur für bestimmte Parameter $(\alpha, \beta)$ sicher und auch nur für eine kleine Auswahl von Parametern kolonnenstabil. Unter Kolonnenstabilität wird verstanden, dass auch eine Kette von mehreren Fahrzeugen, die hintereinander herfahren, nicht anfängt sich aufzuschaukeln: Aus kleinen Störungen, z. B. ein schwaches Bremsen des ersten Fahrzeugs wird entlang der Kette ein immer stärkeres, das im Extremfall bis zum Stillstand eines der Fahrzeuge in der Kette führen kann. Oder auch zu einem Verkehrsunfall. Dieses Verhalten ist bislang nur in sehr speziellen Situationen gefunden worden (s. z. B. [19]), es scheint nicht der Normalfall zu sein.

Allerdings werden die Parameter mit Kolonnenstabilität von menschlichen Fahrern als nicht sehr angenehm empfunden, weshalb AIC-Regler hier meistens einen Kompromiss eingehen, der zu einer schwachen Kolonneninstabilität führt [12].

Daher wird hier ein anderer Ansatz betrachtet, der in der Tradition der Modelle in [14], [15], [16] steht. In einem ersten Schritt wird überlegt, dass eine wichtige Bedingung für sicheres Fahren erfüllt ist, wenn Folgendes gilt:

$$
d(v)+v \tau \leq D(V)+g .
$$

In dieser Gleichung sind $D(V), d(v)$ die Bremswege des führenden und des folgenden Fahrzeugs. Offensichtlich setzt dieses Modell voraus, dass der Folgefahrer eine Vorstellung davon hat, ob und wie das Führungsfahrzeug fahren bzw. bremsen wird. Das ist sicherlich nicht vollständig korrekt, dennoch funktioniert Fahren in vielen Fällen unter der Annahme, dass die anderen Fahrer sich so ähnlich verhalten wie man selbst.

Allerdings bedeutet das auch, dass sich der aus dieser und der folgenden Gleichung resultierende Ansatz durch ein ,merkwürdiges“ Verhalten des Führungsfahrzeuges austricksen lässt. Verfügt das Führungsfahrzeug z. B. über ein Notbremssystem, das Verzögerungen bis zu $12 \mathrm{~m} / \mathrm{s}^{2}$ erlaubt, dann verletzt es die Annahme, dass es sich so ähnlich verhält wie das Folgefahrzeug - typische Verzögerungswerte menschlicher Fahrer liegen im Bereich bis maximal $4 \mathrm{~m} / \mathrm{s}^{2}$-, und weist damit einen viel kürzeren Bremsweg auf. Ein wenig lässt sich das noch ausgleichen, wie auch die folgenden Simulationsergebnisse zeigen, weil die aus diesem Ansatz resultierenden Gleichungen bei starkem Bremsen des Führungsfahrzeugs im Extremfall ihre eigene Bremsverzögerung überschreiten können. Andererseits ist 
dieser Ansatz auch einer, den man für die Entwicklung von Fahrermodellen für die Verkehrssicherheit weiter treiben könnte.

Das obige Modell lässt sich weiterentwickeln, indem verlangt wird, dass die Sicherheitsbedingung nicht nur zur aktuellen Zeit $t$ erfüllt sein soll, sondern auch noch eine gewisse Zeit $t+T$ in der Zukunft. Die Zeit $T$ ist dabei die Antizipationszeit, also die Länge des Planungshorizonts des Fahrers. Bezeichnet die Notation $x$ den Wert der Variablen $x$ zur Zeit $t+T$, wird aus der Sicherheitsgleichung:

$$
d\left(v^{\prime}\right)+v^{\prime} \tau \leq D\left(V^{\prime}\right)+g^{\prime} .
$$

Diese Gleichung kann aber nun nach der Beschleunigung $a$ umgestellt werden. So ist $x^{\prime}=x+v T+a T^{2} / 2$, und zusammen mit einem Ansatz für die Bremswege $d(v)=v^{2} /(2 b)$ lässt sich dann die Sicherheitsgleichung nach $a$ auflösen. Dafür gibt es verschiedene Ansätze, hier wird vor allem der exakte Ansatz verfolgt:

$$
\dot{v}=\frac{1}{T}\left(-b(\tau+T / 2)+\sqrt{b^{2}(\tau+T)^{2}+V^{2}+2 b v T+2 b(g+\Delta v T)}-v\right) .
$$

Interessanterweise führt dieser Ansatz für $T \rightarrow 0$ auf den in SUMO [17] verwendeten zurück.

Eine andere Möglichkeit, [15] folgend, ist eine Taylor-Entwicklung von $d\left(v^{\prime}\right)=d(v+a T) \approx d(v)+a T v / b(v)$, was interessanterweise auf eine lineare Gleichung für $a$ führt, die einfacher zu lösen und numerisch weniger komplex ist:

$$
a=\frac{V^{2}-v^{2}+2 b(T \Delta v+g-v \tau)}{T(2 b \tau+b T+2 v)} .
$$

Obwohl diese Gleichungen kompliziert aussehen und es einigermaßen unwahrscheinlich ist, dass Menschen tatsächlich eine Wurzel aus einem komplizierten Ausdruck ziehen können, sieht sie grafisch dem Helly-Modell doch sehr ähnlich. Das ist deshalb interessant, weil es sich in der Tat gut vorstellen lässt, dass ein menschlicher Fahrer in der Lage ist, eine lineare Abwägung etwa nach dem Motto „Ich bin etwas schneller als der vor mir, aber der Abstand ist groß, daher muss ich gegenwärtig nichts ändern“ durchführt. Eine Vorstellung, wie diese Beschleunigungsfunktion für realistisch gewählte Parameter aussieht, vermittelt Abb. 15.2.

In diesem Zusammenhang ist es noch interessant zu wissen, ob dieser Ansatz tatsächlich zusammenstoßfrei ist. Die simple Antwort lautet: nein. Unter bestimmten Bedingungen lässt sich die Dynamik, die aus Gleichung (15.3) folgt, in der Tat austricksen. Das sei an einer Kette von Fahrzeugen demonstriert, die einem Führungsfahrzeug folgen, das nach einem bestimmten Protokoll $a_{0}(t)$ fährt. Dabei lassen sich als wesentliche Parameter in der Dynamik des Führungsfahrzeugs vor allem die maximalen Beschleunigungen parametrieren. Von besonderem Interesse sind dabei die maximalen Bremsverzögerungen und die Frage, ob man bei dem Modell einen Zusammenstoß produzieren kann. 

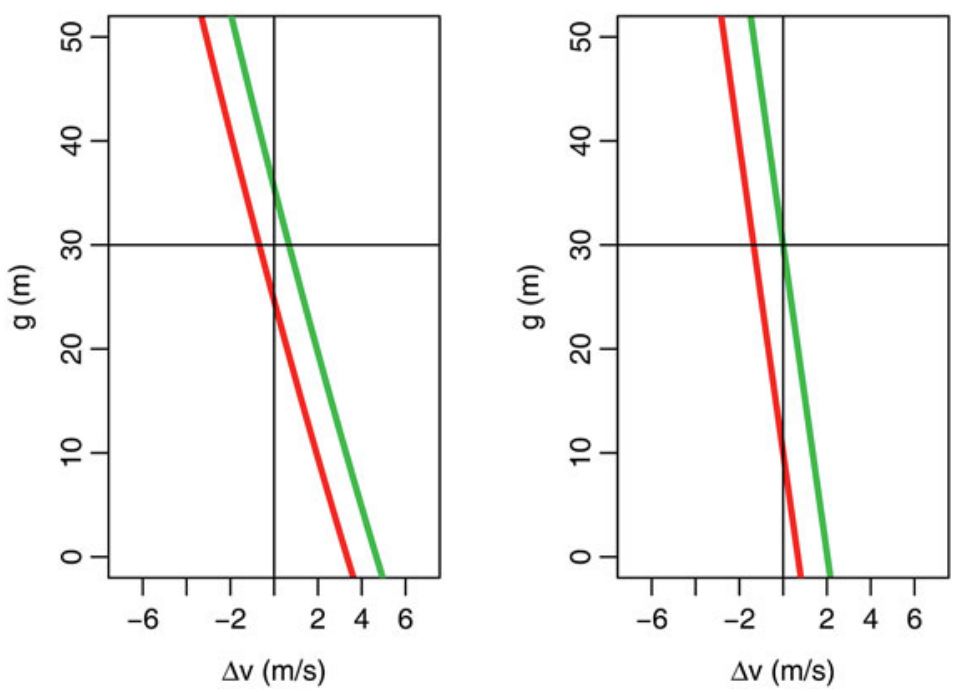

Abb. 15.2 Darstellung der Beschleunigungsfunktionen. Statt hier die gesamte Funktion zu zeichnen, wird nur der durch zwei Linien begrenzte Bereich im $(\Delta v, g)$-Raum dargestellt, in dem die Beschleunigung beider Modelle klein ist. Links von den Linien bremst das Fahrzeug, rechts davon beschleunigt es. Die linke Abbildung ist das Modell von Gleichung (15.3), die rechte das HellyModell (15.2). Die gewählten Parameter sind $V=20, \tau=1,5, b=4, T=2$

Natürlich kann kein Verfahren wirklich alle Möglichkeiten durchtesten. Das folgende Vorgehen ermöglicht aber zumindest eine Abschätzung, wie sicher die Modelle sind. Dazu folgen in einer Simulation $n=50$ Fahrzeuge einem Führungsfahrzeug, das nach einem bestimmten Protokoll seine Beschleunigung wählt. Unter anderem verzögert es immer wieder bis zum Stillstand, zum Teil auch mit Bremsverzögerungen, die an der Grenze der derzeitigen fahrdynamischen Möglichkeiten sind. Aus Untersuchungen zu diesem Thema ergab sich sehr schnell, dass die Modelle nur dann ohne Unfall auskommen, wenn die Antizipationszeit $T$ beim Bremsen auf einen kleineren Wert gesetzt wird. Im Folgenden werden die Modelle immer mit $T=2 \mathrm{~s}$ beim normalen Fahren und mit $T=0,5 \mathrm{~s}$ beim Bremsen betrieben.

Aus den entsprechenden Simulationen geht dann hervor, dass unter diesen Randbedingungen keine Unfälle mit dem Modell in Gleichung (15.3) auftreten, jedenfalls nicht mit dem gewählten Protokoll $a_{0}(t)$. Das Helly-Modell ist allerdings mit den gewählten Parametern nicht so tolerant und produziert gelegentlich Auffahrunfälle.

\subsection{Mensch versus Maschine}

An diesem Punkt stellt sich die Frage, was denn eigentlich einen menschlichen Fahrer von einem autonomen Fahrzeug unterscheidet. Bislang ist nur ein wesentlicher Unterschied zu 


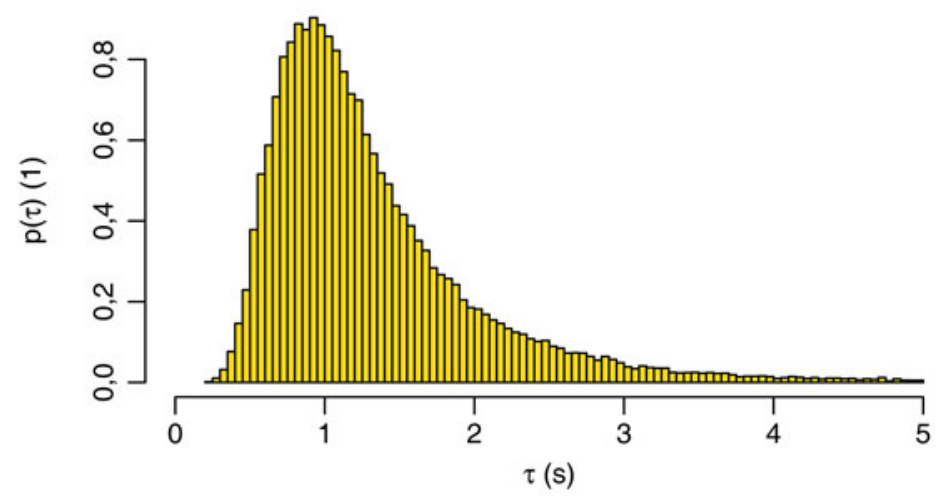

Abb. 15.3 Abstandsverteilung auf der linken Spur der A3. Dargestellt ist die Dichte der jeweiligen Zeitlücke. Ziemlich genau bei 1,1 s liegt das Maximum der Funktion, während der Mittelwert bei 1,4 s liegt. Es werden zum Teil gefährlich kurze Zeitlücken beobachtet

erkennen, und das ist die Zeitlücke $\tau$, mit der die beiden fahren. Menschen sollten nicht mit weniger als $\tau=0,9 \mathrm{~s}$ Abstand fahren, die gesetzliche Empfehlung ist sogar $\tau=2 \mathrm{~s}$, eine Maschine kann im Prinzip mit $\tau=0,3 \ldots 0,5 \mathrm{~s}$ Abstand fahren [1]. Eine beispielhafte Auswertung der tatsächlich gefahrenen Abstände (s. Abb. 15.3) auf einer deutschen Autobahn zeigt (im Bereich um $100 \mathrm{~km} / \mathrm{h}$, wo die größten Verkehrsstärken erreicht werden), dass einige (wenige) menschliche Fahrer diesem „Ideal“ nahe kommen, die überwältigende Mehrheit zeigt aber ein gesetzkonformes Verhalten.

Abb. 15.3 demonstriert auch, dass das menschliche Verhalten eine beträchtliche Bandbreite aufweist [18], was im Gegensatz zu autonomen Fahrzeugen steht: Diese würden alle mit einem kleinen und sehr ähnlichem Wert von $\tau$ fahren. Diese Bandbreite lässt sich detaillierter charakterisieren [17] und quantifizieren. Im Wesentlichen kann gesagt werden, dass $\tau$ nicht nur zwischen verschiedenen Fahrern unterschiedlich ist, sondern sogar bei ein und demselben Fahrer nicht konstant bleibt. Leider ist $\tau$ nicht präzise beobachtbar, vor allem dann nicht, wenn das Führungsfahrzeug selbst die Geschwindigkeit permanent verändert, von daher können an dieser Stelle nur Vermutungen angestellt werden, wie sich $\tau$ im Zeitverlauf verändert. Das führt dann auf sogenannte 2-D-Modelle [17], [19], bei denen $\tau$ in jedem Zeitschritt variiert. Eine einfache Vorstellung, die auf eine solche Dynamik führt, ist, dass der Fahrer bei der Bestimmung des Abstandes einen Fehler macht. Allerdings ist dieser Fehler zeitlich korreliert, d. h. wenn zu einem Zeitpunkt der geschätzte Abstand kleiner ist als der wahre Abstand, so wird das auch noch eine gewisse Zeit danach der Fall sein. Und, mit einiger Wahrscheinlichkeit, ist der Fehler asymmetrisch: Abstände werden oft deutlich kürzer abgeschätzt als sie tatsächlich sind. Jedenfalls führt ein solcher Modellierungsansatz auf eine sehr breite Verteilung von $\tau$-Werten, genau wie empirisch beobachtet.

Ein zweiter Punkt, der einen Mensch von einer Maschine unterscheidet, ist der sogenannte Aktionspunktmechanismus [20]. Genau genommen lässt sich ein menschlicher 


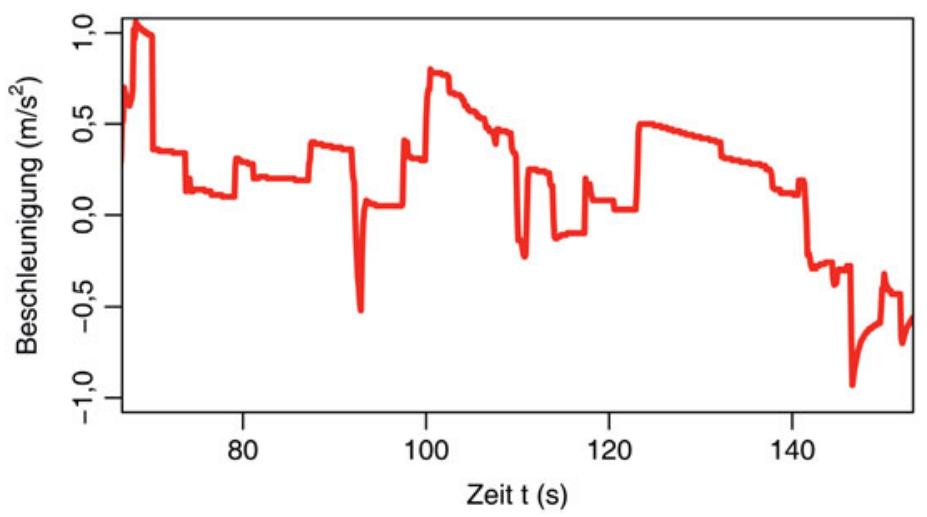

Abb. 15.4 Beschleunigung als Funktion der Zeit bei einem menschlichen Fahrer. Es ist zu sehen, dass sich die Beschleunigung an den sogenannten Aktionspunkten sprunghaft ändert. Zwischen den Aktionspunkten bleibt sie näherungsweise konstant. Die Daten sind bei einer „Fahrt“ des Autors mit einem Fahrsimulator aufgenommen worden, ähnliche Bilder finden sich in allen Datensätzen mit einer ausreichend guten Messung der Beschleunigung oder der Stellung von Gas- und Bremspedal

Fahrer nicht durch eine Differenzialgleichung (15.1) beschreiben. Vielmehr erfolgt die Kontrolle eines Fahrzeugs durch Korrektur der Beschleunigung (Gaspedalstellung) in unregelmäßigen Zeitabständen, ein Beispiel dafür zeigt Abb. 15.4.

Die zeitlichen Abstände zwischen aufeinanderfolgenden Aktionspunkten folgen ebenfalls einer sehr breiten Verteilung mit Werten zwischen 0,5 und 1,5 s. Offensichtlich ist hier ein weiterer Modellierungsansatz für Verkehrssicherheitsfragen - wenn die Zeit zwischen zwei Aktionspunkten sehr lang wird, dann kann es zu einer kritischen Situation kommen. Im Normalfall passiert das aber nicht, und dann gibt es nur geringe Unterschiede zwischen einer Modellierung nach Gleichung (15.1) und einer Modellierung, in der die Aktionspunkte explizit verwendet werden [21]. Insbesondere führt der Aktionspunktmechanismus allein nicht zu einer breiten Verteilung von Abständen zwischen den Fahrzeugen.

Auch dieses sei wieder am Beispiel der in Abschn. 15.2 benutzten Kette von Fahrzeugen demonstriert, die einem Führungsfahrzeug folgen. Eine Auswertung des (in der Simulation) gemessenen Abstandes, hier als Funktion der Nummer des Folgefahrzeuges, zeigt, dass ein autonomes Fahrzeug in den meisten Fällen mit deutlich geringerer Varianz dem Führungsfahrzeug folgt - trotz dessen zum Teil sehr volatilen Verhaltens. Eine Darstellung dazu findet sich in Abb. 15.5.

Somit sind die in diesem Kapitel verwendeten Modelle spezifiziert, und der Unterschied zwischen der menschlichen und der autonomen Fahrweise ist charakterisiert. Im Folgenden wird anhand einiger Anwendungen demonstriert, was das für typische Verkehrsmanagementanwendungen bedeutet. 


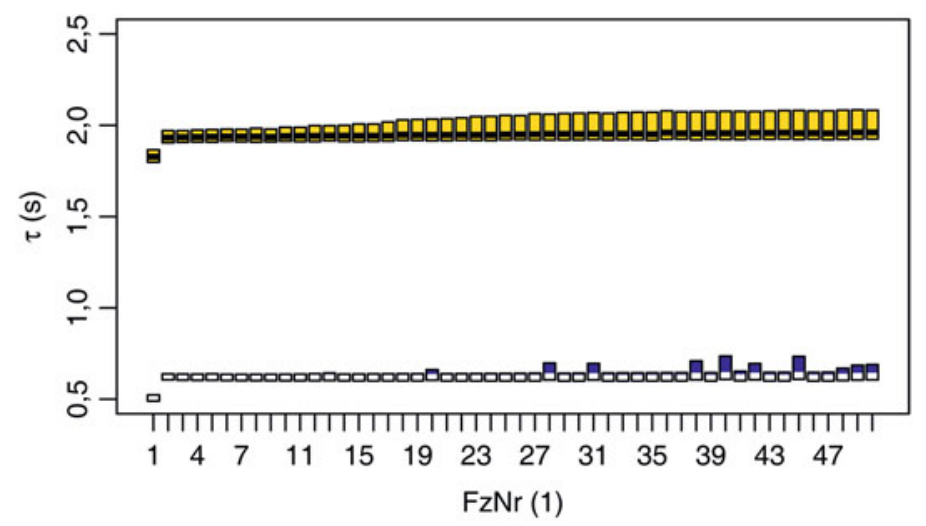

Abb. 15.5 Abstandsverhalten für menschliche und autonome Fahrzeuge. Dargestellt ist der Median des Abstands und die 25 Prozent- und 75 Prozent-Perzentile, jeweils als Funktion der Position in der Kette. Die obere Kurve zeigt das Modell des menschlichen Fahrers, die untere modelliert eine Kette von autonomen Fahrzeugen

\subsection{Anfahren an einer Lichtsignalanlage (LSA)}

Dieser Prozess ist eine der Situationen, bei dem autonome Fahrzeuge erhebliche Gewinne versprechen. An einem Zufluss einer LSA wird im Folgenden die Verlustzeit pro Fahrzeug für eine beliebige Kombination aus normalen und autonomen Fahrzeugen untersucht. Dabei bezeichnet $\eta$ den Anteil autonom fahrender Fahrzeuge, mit der Annahme $\tau=0,5 \mathrm{~s}$ für autonome und $\tau=1,5 \mathrm{~s}$ für normale Fahrzeuge. Die Simulationsergebnisse werden darüber hinaus durch eine theoretische Betrachtung gestützt. Für die beschriebene Situation gibt es eine solche Theorie, die in [22] entwickelt wurde. Interessanterweise lässt sie sich auf eine Situation mit einem Mix aus autonomen und normalen Fahrzeugen übertragen. Dann lautet der entsprechende Ausdruck:

$$
d(q, \eta)=\frac{c}{2} \frac{(1-\lambda)^{2}}{1-\mathrm{y}}+\frac{1}{2} \frac{x^{2}}{\mathrm{q}(1-\mathrm{x})}, \lambda=\frac{g}{c}, y=\frac{q}{s}, x=\frac{y}{\lambda}, s=s_{0}(1-\eta)+s_{1} \eta
$$

In Gleichung (15.4) ist $q$ die Nachfrage, $s_{0}$ die Kapazität eines Stroms von Menschen gesteuerter Fahrzeuge, $s_{1}$ die Kapazität eines Stroms automatisierter Fahrzeuge, $g$ die Freigabezeit (Grünzeit) und $c$ die Umlaufzeit der Anlage. Die Umlaufzeit ist die Zeit, die vergeht, bis die Anlage wieder den Anfangszustand erreicht hat. Die Simulationsergebnisse für ausgewählte Variationen der Nachfrage $q$ und des Anteils autonomer Fahrzeuge $\eta$ ist in Abb. 15.6 dargestellt.

Die Kurven in Abb. 15.6 wurden aufgenommen, indem verschiedene Werte der Nachfrage $q$ (variierend von $18 \mathrm{Fz} / \mathrm{h}$ bis $1800 \mathrm{Fz} / \mathrm{h}$ ) für jeweils $5 \mathrm{Stunden}$ simuliert wurden. Die Nachfrage selbst ist eine stochastische Größe (näherungsweise Poisson-verteilt), d.h., in jedem betrachteten Zeitintervall kommt immer eine andere Anzahl von Fahrzeugen an, 


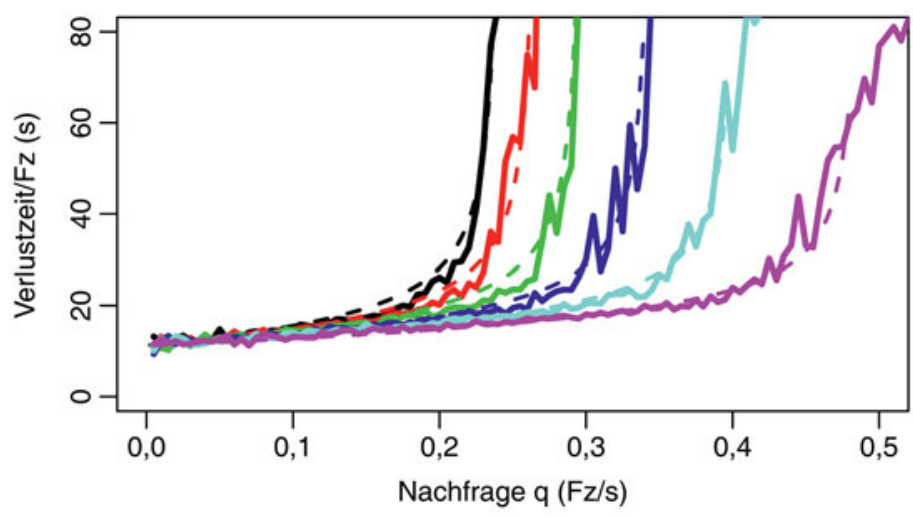

Abb. 15.6 Verlustzeit an einer LSA als Funktion der Nachfrage und für verschiedene Ausstattungsraten $\eta=0,20 \%, 40 \%, 60 \%, 80 \%$ und $100 \%$ (von links nach rechts). Die gestrichelten Linien sind aus Gleichung (15.4) berechnet, allerdings mit einer Kapazität $s$, die direkt in der Simulation gemessen wurde

nur der Mittelwert über viele solcher Zeitintervalle ergibt dann die korrekte (gesetzte) Nachfrage.

Für jedes simulierte Fahrzeug wurde die Verlustzeit aufgezeichnet und aus diesen Verlustzeiten der in Abb. 15.6 eingetragene Mittelwert ausgerechnet. Im Prinzip kann die ganze Verteilung der Verlustzeiten zur Charakterisierung der Ergebnisse herangezogen werden, was aus Platzgründen unterbleibt, obwohl es interessant wäre: Die Schwankungen der Verlustzeiten sind ein Maß für die Verlässlichkeit eines solchen Systems. Allerdings erweist sich in dem betrachteten Beispiel, dass die Schwankungen der Verlustzeit nur sehr schwach vom Anteil der autonom fahrenden Fahrzeuge abhängt, die wesentliche Quelle von Stochastizität in diesem System wird von der Nachfrage generiert und nicht von der Dynamik der Fahrzeuge.

Zwei Ergebnisse fallen in Abb. 15.6 auf. Zum einen stimmt die Beschreibung durch die Theorie nicht immer mit den Simulationsergebnissen überein. Hier steckt noch einiges an Forschungsbedarf, weil es gar nicht so einfach ist, die in der Theorie vorausgesetzten Annahmen in die simulative Realität zu übertragen. Das wird sicherlich beim Vergleich mit realen Messwerten noch einmal schwieriger. Für die erzielte Übereinstimmung mussten darüber hinaus aus der Simulation ermittelte Werte für die Sättigungsverkehrsstärke verwendet werden - mit den theoretischen Werten, also den $\tau$-Werten wie in Abschn. 15.3 definiert, ist die Übereinstimmung nicht überzeugend.

Zum anderen verändert sich durch die autonomen Fahrzeuge „nur“ die Kapazität, ansonsten gibt es keine weiteren oder nur sehr geringe Gewinne. Solange die Nachfrage von der jeweiligen Kapazität entfernt ist, gibt es auch nur geringe Unterschiede zwischen den verschiedenen Szenarien, zumindest nicht auf der Ebene der hier gewählten Beschreibung.

Eine Änderung der Kapazität hat allerdings einen sehr positiven Effekt: Es bedeutet, dass die notwendigen Freigabezeiten an einer LSA kürzer werden können und damit mehr Zeit für andere Verkehrsträger zur Verfügung steht. 


\subsection{Adaptive Lichtsignalanlage}

In Abschn. 15.4 wurde eine Lichtsignalanlage betrachtet, die in Festzeitsteuerung läuft. Viele moderne Anlagen laufen allerdings mit einer adaptiven Steuerung. Das bedeutet, dass die LSA versucht, ihre Freigabezeiten nach der aktuellen Nachfrage einzurichten. Bei kleiner Nachfrage resultieren daraus kurze Freigabezeiten, bei großer Nachfrage reagiert die Anlage mit langen Freigabezeiten. Die Details sind etwas komplizierter, weil die Verlustzeit als Funktion der Nachfrage betrachtet ein Minimum bei einer bestimmten optimalen Umlaufzeit hat. Eine adaptive Anlage ist in der Lage, sich die optimale Umlaufzeit selbst auszusuchen, darüber hinaus nutzt sie auf sehr geschickte Weise die Schwankungen aus, die in einem Verkehrsstrom vorkommen.

Auch in diesem Fall soll untersucht werden, wie eine solche adaptive Anlage mit einem Gemisch aus autonomen und normalen Fahrzeugen umgeht. Zu diesem Zweck wurde die Simulation einer zweiarmigen Kreuzung aufgesetzt, die mit einem adaptiven Verfahren gesteuert wird [25]. Die beiden Arme sind $600 \mathrm{~m}$ lang, gemessen wird wieder die Verlustzeit pro Fahrzeug an dieser Kreuzung. Im Unterschied zu Abschn. 15.4 wurde jetzt aber eine Nachfrage gewählt, die von der Zeit abhängt und damit eine typische Spitzenstundengruppe nachbildet, bei der zur Zeit des Nachfragemaximums die Anlage trotz ihrer Adaptivität gesättigt ist. Die hier gewählte Nachfragefunktion lautet:

$$
q(t)=q_{0}+q_{1} \sin \left(\frac{\pi t}{T}\right)
$$

wobei $q_{0}$ eine Grundlast ist, $q_{1}$ die Amplitude der Nachfrageschwankung und $T$ die gesamte Zeitdauer der Simulation. Beide Arme werden mit der gleichen Nachfrage belastet, was einen relativ ungünstigen Fall darstellt.

Neben den Verlustzeiten sind in diesem Fall vor allem die Freigabezeiten von Interesse. Da die Anlage diese Zeiten der Nachfrage anpasst, schwanken diese innerhalb typischer Grenzen. In vielen Ländern kann die Freigabezeit nicht beliebig schwanken: So kann die Freigabezeit bei einer normalen Lichtsignalanlage nicht unter $5 \mathrm{~s}$ sinken, und in den folgenden Simulationen ist die maximale Freigabezeit auf $40 \mathrm{~s}$ gesetzt.

Eine solche Simulation ist auch ein interessanter Fall bei der Auswertung der Simulationsdaten. Eine einzelne Simulation einer solchen Spitzenstunde weist starke Schwankungen sowohl bei den Verlustzeiten als auch bei den Freigabe- und Umlaufzeiten auf. Zwar wurden die Verlustzeiten jeweils über einen Umlauf der Anlage gemittelt, das reicht aber nicht, weil diese Umläufe selbst eine stochastische Größe sind, deren Mittelwert und Statistik sich erst aus genügend häufigen Wiederholungen desselben Szenarios, aber mit leicht verschiedenen Details ergibt - ganz so wie in der Realität, wenn aufeinanderfolgende Tage betrachtet werden. Um zu statistisch validen Ergebnissen zu kommen, wurde in diesem Fall die Spitzenstunde 50-mal wiederholt. Jeweils in Fünf-Minuten-Intervallen wurden die Mittelwerte der Verlustzeiten über den letzten Umlauf und die dazu gehörende, von der Anlage eingestellte Freigabezeit gesammelt. Aus diesen Daten setzen sich dann die Ergebnisse in Abb. 15.7 zusammen. 

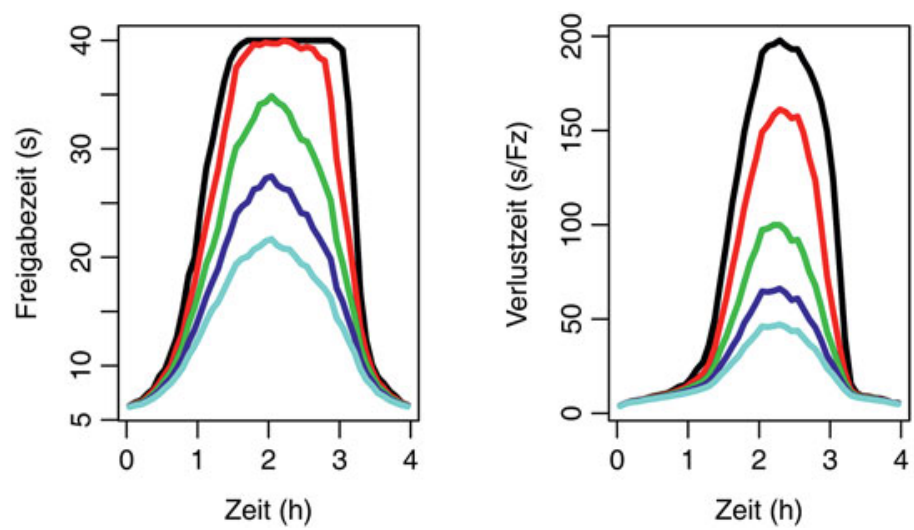

Abb. 15.7 Freigabezeiten (links) und Verlustzeiten (rechts) an einer simulierten adaptiven Anlage, dargestellt als Funktion der Zeit und für verschiedene Anteile autonomer Fahrzeuge, $\eta=0 \%, 25 \%$, $50 \%, 75 \%, 100 \%$. Die Nachfrage war mit $q_{0}=180 \mathrm{Fz} / \mathrm{h}$ und $q_{1}=720 \mathrm{Fz} / \mathrm{h}$ parametriert

Bei der maximalen Nachfrage verlängert die Anlage die Freigabezeiten bis zu der Grenze von 40 Sekunden und zeigt damit an, dass sie tatsächlich ihre Sättigung erreicht hat. Allerdings gilt das nur für einen Strom normaler Fahrzeuge. Sobald autonome Fahrzeuge hinzukommen, sinkt der Spitzenwert bei der Verlustzeit, und bei einer Ausstattungsrate von 50 Prozent wird nicht einmal mehr die maximale Freigabezeit erreicht. Das entspricht der Beobachtung in Abschn. 15.4, dass autonome Fahrzeuge nicht nur die Kapazität erhöhen, sondern auch zu einer Reduktion der Freigabezeiten beitragen - ein Effekt, der in diesem Beispiel hier ziemlich deutlich ist, schon ein kleiner Anteil autonom fahrender Fahrzeuge kann sich hier bemerkbar machen.

\subsection{Grüne Welle mit autonomen Fahrzeugen}

Die bisherigen Szenarien haben eine Kreuzung untersucht. Sehr viel interessanter ist der Fall eines Streckenabschnitts mit mehreren Kreuzungen hintereinander, die alle von einer LSA geregelt werden. In diesem Fall spielt die Koordinierung zwischen den Anlagen, umgangssprachlich als „grüne Welle“ bezeichnet, eine wichtige Rolle. Auch hier wird mithilfe einer Simulation untersucht, wie groß die Auswirkungen der Einführung von autonomen Fahrzeugen sind. Analog dem Vorgehen in [26] wird eine Strecke mit zehn Kreuzungen simuliert und dabei die Koordinierung verändert. Unverändert bleiben die Nachfrage, die konstant ist, die Grünzeiten und die Umlaufzeiten. Verändert wird lediglich der Offset, also der Zeitpunkt, zu dem die Anlage den Fahrzeugstrom in Fahrtrichtung freigibt. Wenn dieser Zeitversatz zwischen zwei Anlagen gerade der Reisezeit zwischen den beiden Anlagen entspricht, ist das System in seinem optimalen Zustand: Die Verlustzeit der Fahrzeuge an der stromabwärtigen Anlage ist exakt null, wenn die beiden Freigabe- 


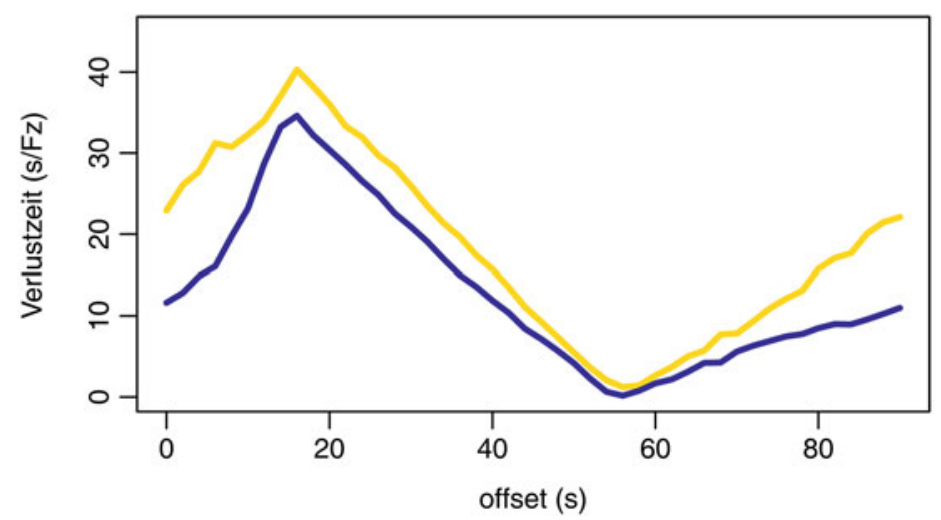

Abb. 15.8 Verlustzeit als Funktion der Versatzzeit für eine einfache grüne Welle. Dargestellt ist hier eine Simulation mit menschlichen Fahrern (gelbe Kurve) und eine nur mit autonomen Fahrzeugen (blaue Kurve). Gezeigt ist hier das beste Ergebnis, das zwischen der ersten und der zweiten Kreuzung erreicht wird

zeiten gleich sind. Dann ist einsichtig, dass genauso viele Fahrzeuge die Kreuzung passieren können, wie von der Anlage stromaufwärts losfahren.

Es lässt sich schon ahnen: In diesem Fall sind mit autonomen Fahrzeugen keine Verbesserungen zu erzielen, und genau das demonstriert das Simulationsergebnis in Abb. 15.8. Allerdings verbessern autonome Fahrzeuge im Falle von nicht-optimaler Koordinierung dann doch die Verlustzeiten. Das liegt daran, dass der Pulk von Fahrzeugen, der eine Anlage verlässt, stärker komprimiert ist als im Fall der menschlichen Fahrer.

\subsection{Simulation einer Stadt}

Im letzten Abschnitt soll untersucht werden, wie sich die Einführung autonomer Fahrzeuge in einer ganzen Stadt auswirken könnte. Zu diesem Zweck wird eine bestehende SUMOSimulation [17], [23] der Stadt Braunschweig verwendet, um den Einfluss autonomer Fahrzeuge auf den Verkehrsablauf eines Verkehrssystems bewerten zu können.

Allerdings ist in SUMO das in Abschn. 15.2 eingeführte Modell nicht implementiert, sodass die Simulation mit den Modellen auskommen muss, die in SUMO verfügbar sind. Verwendet wird daher das in SUMO eingebaute Standardmodell, das bei der Beschreibung der Schwankungen der Fahrer nicht so ausgefeilt ist wie das hier eingeführte Modell.

Um das Modell aufzusetzen, wird ein modifiziertes Netz der Firma NavTeq verwendet, in Abb. 15.9 ist ein Ausschnitt des entsprechenden Verkehrsnetzes zu sehen, die ganze Simulation umfasst die gesamte Fläche der Stadt Braunschweig einschließlich der umgebenden Autobahnen. Insgesamt enthält das Simulationsnetz rund 129.000 Kanten.

Die notwendige Nachfrage nach Verkehr stammt aus einer Start/Ziel-Matrix der Firma ptv, die für verschiedene Tage der Woche und für jeden dieser Tage in 24 Zeitscheiben von 


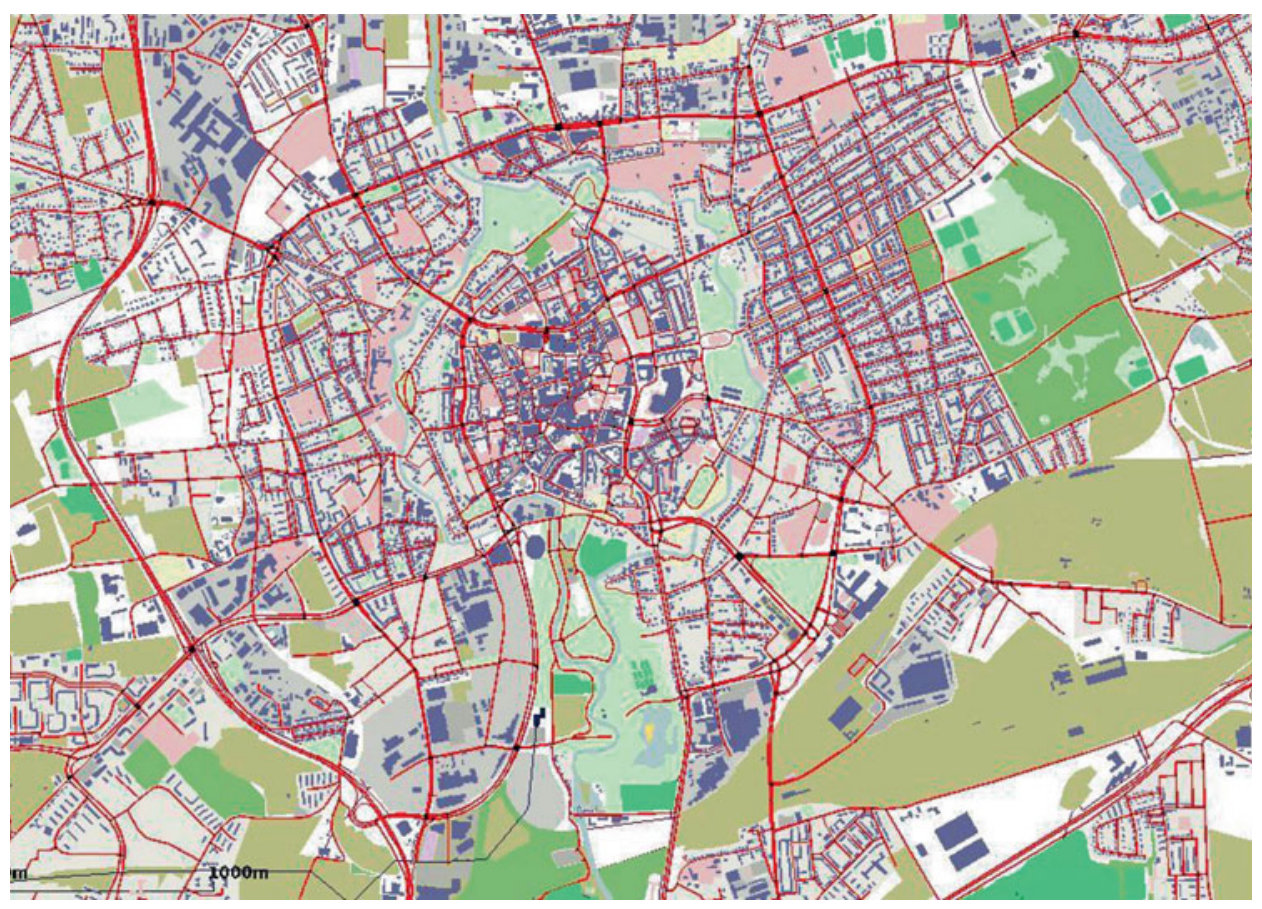

Abb. 15.9 Ausschnitt aus dem Simulationsnetz der Stadt Braunschweig. Die Flächennutzungsdaten stammen aus der OpenStreetMap-Datenbasis [24]

je einer Stunde Dauer vorliegt. Mit der Nachfrage wurde ein sogenanntes Nutzergleichgewicht ausgerechnet, das in diesem Fall rund 100 Iterationsschritte erforderte. Am Ende dieses Prozesses steht für jedes Fahrzeug, das in SUMO simuliert wird, eine Route, die optimal ist in dem Sinn, dass jede andere Route durch das Netz länger dauert. Insgesamt werden 647.000 Fahrzeuge simuliert. Erste Vergleiche mit realen Zähldaten aus Braunschweig deuten darauf hin, dass die Matrix die Nachfrage deutlich unterschätzt. Das hat sicherlich Auswirkungen auf die hier diskutierten Ergebnisse, allerdings konnten im Rahmen dieses Projektes solche Korrekturen nicht mehr umgesetzt werden.

Um autonome Fahrzeuge simulieren zu können, wird ein neuer Fahrzeugtyp eingeführt, der so ähnlich parametriert ist wie die Modelle in Abschn. 15.2: Die autonomen Fahrzeuge in SUMO fahren mit $\tau=0,5 \mathrm{~s}$, alle anderen mit $\tau=1$ und $\sigma=0,5$ Dabei ist $\sigma$ der Rauschparameter in SUMO, welcher vorgibt, um wie viel ein Fahrzeug von der optimalen Fahrweise abweicht. Die Wahl von $\tau=0,5 \mathrm{~s}$ führt dazu, dass auch die Schrittweite in SUMO auf 0,5 s gesetzt werden muss, damit die Fahrzeuge weiterhin ohne Zusammenstöße fahren können. Damit verlängert sich die Simulationszeit von rund $50 \mathrm{~min}$ auf $90 \mathrm{~min}$ für die Simulation eines ganzen Tages in Braunschweig.

Nur die Pkw wurden als autonome Fahrzeuge simuliert: Die rund 44.000 Lkw blieben unverändert. Ebenfalls nicht vollständig korrekt abgebildet sind in dieser Simulation die 


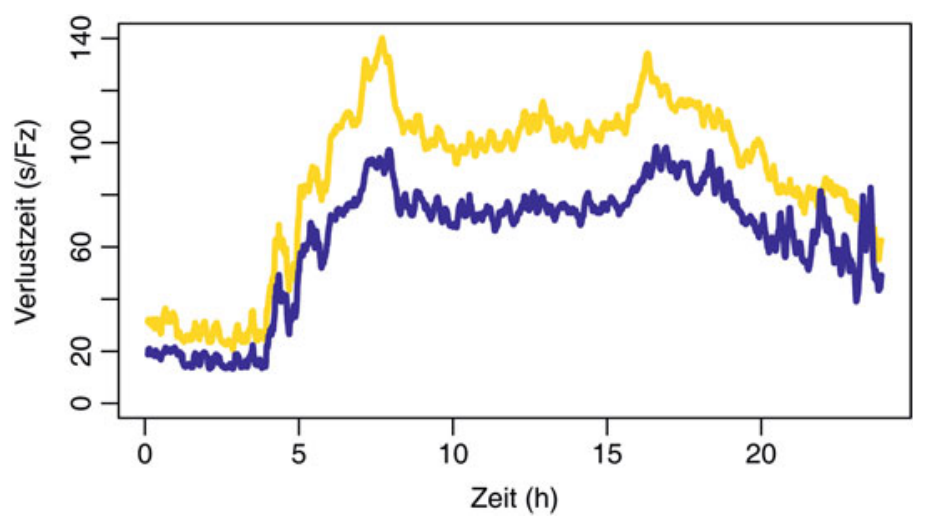

Abb. 15.10 Vergleich der Verlustzeiten zwischen einer Simulation mit menschlichen Fahrern (gelbe Kurve) und einer Simulation, bei der die Pkw autonom fahren (blaue Kurve). Jeder Datenpunkt ist ein gleitender Durchschnittswert aus den acht benachbarten Ein-Minuten-Werten. Die Streuung der Werte beider Kurven ist nicht sehr unterschiedlich und deshalb nicht dargestellt

Lichtsignalanlagen. Somit ist davon auszugehen, dass auch von dieser Seite weitere Korrekturen des unten stehenden Simulationsergebnisses zu erwarten sind.

Trotzdem liefert diese Simulation erste wichtige Erkenntnisse, die in Abb. $15.10 \mathrm{zu}$ sehen sind. Selbst ohne weitere Maßnahmen ist das autonome System in dem Sinne effizienter, dass zwischen fünf und 80 Prozent Verlustzeit eingespart wird, mit einem Mittelwert von rund 40 Prozent. Mit den gewählten Parametern ändert sich allerdings die Varianz der Reisezeiten relativ wenig, das System wird somit zwar schneller, aber nicht unbedingt auch verlässlicher. Das könnte sich ändern, wenn auch das Verkehrsmanagement realistisch simuliert wird, entsprechende Arbeiten werden derzeit vorbereitet.

\subsection{Fazit}

In diesem Beitrag wurden erste Überlegungen angestellt, wie ein Verkehrsmanagement auf die Möglichkeiten des autonomen Fahrens eingestellt werden muss. Die präsentierten Fallbeispiele demonstrieren, dass je nach Szenario ganz verschiedene Verbesserungen im Verkehrsablauf durch das Einführen autonomer Fahrzeuge zu erreichen sind.

Leider lassen sich die zu erreichenden Verbesserungen schwer in eine einzige Zahl zusammenfassen. So wurde beispielsweise in Abschn. 15.4 demonstriert, dass die Kapazität einer LSA sich durchaus verdoppeln lässt. Ist an der entsprechenden Anlage die Nachfrage gering, so ist von dieser Verdoppelung relativ wenig zu merken. Arbeitet die Anlage hingegen am Rande ihrer Kapazität, dann führt schon eine geringfügige Erhöhung der Kapazität zu einer erheblichen Verbesserung.

Das lässt sich sehr schön anhand des Szenarios in Abschn. 15.5 nachvollziehen: Hier durchläuft die Nachfrage alle Werte von sehr gering bis zur (zeitweisen) Übersättigung. 
Während bei kleiner Nachfrage sowohl die Freigabezeiten als auch die Verlustzeiten sich relativ wenig mit dem Einführen autonomer Fahrzeuge ändern, erreicht das System jenseits der Kapazität enorme Verbesserungen. Allerdings ist der Umfang dieser Verbesserungen wieder von den Details des betrachteten Szenarios abhängig. Wäre der Spitzenwert der Nachfrage nur ein wenig kleiner gewesen, dann wäre auch der Gewinn deutlich kleiner geworden.

Dennoch bleibt festzuhalten, dass zumindest im urbanen Kontext das Einführen autonomer Fahrzeuge das Potenzial hat, an den Lichtsignalanlagen deutlich Zeit zu gewinnen, die dann anderen Verkehrsteilnehmern zur Verfügung steht - wenn das Einführen dieser Fahrzeuge nicht zu einer Erhöhung der Nachfrage nach automobilem Transport nach sich zieht.

\section{Literatur}

1. Winner, H.: Private Korrespondenz. (2014)

2. van Dijke, J., van Schijndel, M., Nashashibi, F., de la Fortelle, A.: Certification of Automated Transport Systems. Procedia - Social and Behavioral Sciences 48, 3461-3470 (2012)

3. Kesting, A.: Microscopic Modeling of Human and Automated Driving: Towards Traffic-Adaptive Cruise Control, Verlag Dr. Müller, Saarbrücken, ISBN 978-3-639-05859-8 (2008)

4. Reuschel, A.: Fahrzeugbewegung in der Kolonne bei gleichförmig beschleunigtem oder verzögertem Leitfahrzeug. Zeitschrift des österreichischen Ingenieur und Architektenvereins, 7/8, 95-98 (1950)

5. Chowdhury, D., Santen, L., Schadschneider, A.: Statistical physics of vehicular traffic and some related systems. Physics Reports 329, 199-329 (2000)

6. Helbing, D.: Traffic and Related Self-Driven Many-Particle Systems. Reviews of Modern Physics 73, 1067-1141 (2001)

7. Nagel, K., Wagner, P., Woesler, R.: Still flowing: approaches to traffic flow and traffic jam modelling. Operations Research 51, 681-710 (2003)

8. Treiber, M., Kesting, A.: Traffic Flow Dynamics: Data, Models and Simulation. (2012)

9. Urmson C., et al: Autonomous Driving in Urban Environments: Boss and the Urban Challenge. Journal of Field Robotics 25, 425-466 (2008)

10. Levinson, J. et al.: Towards fully autonomous driving: Systems and algorithms. In proceedings of the 2011 IEEE Intelligent Vehicles Symposium, 163-168 (2011)

11. Campbell M., Egerstedt, M., How, J. P., Murray, R. M.: Autonomous driving in urban environments: approaches, lessons and challenges. Philosophical Transactions of the Royal Society A 368, 4649-4672 (2010)

12. Winner, H., Hakuli, S., Wolf, G.: Handbuch Fahrerassistenzsysteme: Grundlagen, Komponenten und Systeme für aktive Sicherheit und Komfort (2011)

13. Helly, W.: Simulation of bottlenecks in single lane traffic flow. Proceedings of the symposium on theory of traffic flow (1959)

14. Gipps, P.: A behavioural car-following model for computer simulation. Transportation Research Part B 15, 105-111 (1981)

15. Krauß, S.: Microscopic modelling of traffic flow: Investigation of Collision Free Vehicle Dynamics, Dissertation, Universität zu Köln (1998)

16. Krauß, S., Wagner, P., Gawron, C.: Metastable states in a microscopic model of traffic flow. Physical Review E 55, 5597-5602 (1997) 
17. Krajzewicz, D, Erdmann, J., Behrisch, M, Bieker, L.: Recent Development and Applications of SUMO - Simulation of Urban MObility. International Journal On Advances in Systems and Measurements, 5, 128-138 (2012)

18. Wagner, P.: Analyzing fluctuations in car-following. Transportation Research Part B 46, 1384$1392(2012)$

19. Jiang, R., Hu, M., Zhang, H.M., Gao, Z., Jia, B., Wu, Q., Wang, B., Yang, M.: Traffic Experiment Reveals the Nature of Car-Following. PLoS ONE 9: e94351. doi:10.1371/journal.pone.0094351 (2014)

20. Todosiev, E.P., L. C. Barbosa, L.C.: A proposed model for the driver-vehicle-system. Traffic Engineering, 34, 17-20, (1963/64)

21. Wagner, P.: A time-discrete harmonic oscillator model of human car-following. European Physical Journal B 84, 713-718 (2011)

22. Webster, F.V.: Traffic Signal Settings. Department of Scientific And Industrial Research Road Research Laboratory, (1958)

23. Krajzewicz, D., Furian, N., Tomàs Vergés, J.: Großflächige Simulation von Verkehrsmanagementansätzen zur Reduktion von Schadstoffemissionen. 24. Verkehrswissenschaftliche Tage Dresden, Deutschland (2014)

24. OpenStreetMap: www.openstreetmap.org, letzter Zugriff am 29.7.2014

25. Oertel, R., Wagner, P.: Delay-Time Actuated Traffic Signal Control for an Isolated Intersection. In: Proceedings 90th Annual Meeting Transportation Research Board (TRB) (2011)

26. Gartner, N.H., Wagner, P.: Traffic flow characteristics on signalized arterials. Transportation Research Records 1883, 94-100 (2004) 